\title{
Quantification of uncertainties of water vapour column retrievals using future instruments
}

\author{
H. Diedrich, R. Preusker, R. Lindstrot, and J. Fischer \\ Institut für Weltraumwissenschaften, Freie Universität Berlin, Carl-Heinrich-Becker-Weg 6-10, 12165 Berlin, Germany \\ Correspondence to: H. Diedrich (hannes.diedrich@wew.fu-berlin.de)
}

Received: 12 July 2012 - Published in Atmos. Meas. Tech. Discuss.: 6 September 2012

Revised: 15 January 2013 - Accepted: 21 January 2013 - Published: 14 February 2013

\begin{abstract}
This study presents a quantification of uncertainties of total column water vapour retrievals based on simulated near-infrared measurements of upcoming instruments. The concepts of three scheduled spectrometers were taken into account: OLCI (Ocean and Land Color Instrument) on Sentinel-3, METimage on an EPS-SG (EUMETSAT Polar System - Second Generation) satellite and FCI (Flexible Combined Imager) on MTG (Meteosat Third Generation). Optimal estimation theory was used to estimate the error of a hypothetical total water vapour column retrieval for 27 different atmospheric cases. The errors range from $100 \%$ in very dry cases to $2 \%$ in humid cases with a very high surface albedo. Generally, the absolute uncertainties increase with higher water vapour column content due to $\mathrm{H}_{2} \mathrm{O}$-saturation and decrease with a brighter surface albedo. Uncertainties increase with higher aerosol optical thickness, apart from very dark cases. Overall, the METimage channel setting enables the most accurate retrievals. The retrieval using the MTGFCI build-up has the highest uncertainties apart from very bright cases.
\end{abstract}

On the one hand, a retrieval using two absorption channels increases the accuracy, in some cases by one order of magnitude, in comparison to a retrieval using just one absorption channel. On the other hand, a retrieval using three absorption channels has no significant advantage over a two-absorption channel retrieval.

Furthermore, the optimal position of the absorption channels was determined using the concept of the "information content". For a single channel retrieval, a channel at 900 or $915 \mathrm{~nm}$ has the highest mean information content over all cases. The second absorption channel is ideally weakly correlated with the first one, and therefore positioned at $935 \mathrm{~nm}$, in a region with stronger water vapour absorption.

\section{Introduction}

Water vapour is the most important greenhouse gas in the Earth's atmosphere and has a strong connection to various feedback mechanisms on climate (IPCC, 2007). Detecting local and global trends in the water vapour field is therefore an important task for climate research.

Satellite remote sensing can provide high spatial and temporal resolutions. Water vapour detection over ocean surfaces has been done since the 1980s with microwave radiometers (e.g. Schluessel and Emery, 1990). However, the detection of the total water vapour column (TCWV) over land surfaces is much more complicated. Although there are many absorption features of water vapour over the whole electromagnetic spectrum, just a few of them are suited for the retrieval of total water vapour columns. Some existing water vapour retrieval schemes use radiation measurements in the visible (VIS) (Noël et al., 2002) and infrared (IR) (Susskind et al., 2003). This work focuses on concepts of retrievals which analyse measured radiation in the near-infrared (NIR) between $800 \mathrm{~nm}$ and $1000 \mathrm{~nm}$. There are several reasons for that, e.g the influence of Rayleigh scattering and scattering on atmospheric particles, and the spectral dependency of the surface reflectance are comparably low. Above this range, saturation effects are too dominant to retrieve high water vapour columns. Below that range, the absorption lines are too narrow and the sensitivity of the transmittance with respect to water vapour is too low. Although well known above the ocean, the emissivities of land surfaces in the microwave and IR spectrum are highly heterogeneous and mostly unknown. Alternatively, in the VIS and NIR wavelength range, one can estimate the characteristics of the lower boundary (surface reflectance) from top-of-atmosphere 
(TOA) measurements. Consequently, this work only considers the wavelength range of NIR.

There are already several instruments and retrievals which operationally retrieve water vapour over cloud-free land surfaces in the NIR, for example MERIS (MEdium Resolution Imaging Spectrometer) on ENVISAT (Lindstrot et al., 2012; Fischer and Leinweber, 2010). The existing retrievals are various but use a basic principle, the differential absorption technique (Albert et al., 2001, 2005; Bartsch et al., 1996; Gao et al., 1993). The comparison of radiances in a window channel with radiances in an absorption channel provides information about the TCWV.

However, there are a number of error sources for the retrieval of water vapour from NIR-measurements: one part of the uncertainty is due to technical constraints, such as instrumental noise or spectral and absolute calibration of the spectrometers. On top of that, the retrieval is based on various assumptions with respect to, e.g. scattering in the atmosphere or the vertical temperature profile. One goal of this study is the quantification of uncertainties of a hypothetical water vapour retrieval using three future concepts of spectrometers. We selected only concepts of image sensors whose plans include water vapour absorption channels in the NIR. Additionally, we aim to find out how to improve the accuracy of such retrieval by determining the optimal channel setting for the water vapour remote sensing. Although there is no information about the measurement accuracies and the actual retrieval, one can estimate the uncertainties using the optimal estimation theory and the concept of information content.

\section{Instruments}

\subsection{OLCI}

The Ocean and Land Color Instrument (OLCI) is planned to be the sequel of MERIS (Medium Resolution Imaging Spectrometer). It will be installed on the Sentinel-3 orbiter, which is part of the GMES (Global Monitoring for Environment and Security) program. It is scheduled to be launched in 2014 at the earliest with a lifetime of approximately more than $12 \mathrm{yr}$ (fuel for five more years is going to be available). To provide a better temporal resolution, it is planned to operate two identical platforms which orbit the Earth on sun-synchronous tracks at about $815 \mathrm{~km}$ height with a delay of 180 degrees. OLCI will be comprised of five identical cameras in a fan shape alignment (role model: MERIS). It will measure the radiation reflected from the Earth in the visible and nearinfrared spectrum. The spatial resolution of the spectrometer will be $300 \mathrm{~m}$ (full resolution), which can be downscaled to reduced resolution $(1200 \mathrm{~m})$, and will be able to sample a line of $1300 \mathrm{~km}$ (field of view: $68.5^{\circ}$ ) at once. The observation geometry will be tilted by 12 degrees to reduce sun glint effects. With a two-satellite composition, global coverage will be achieved within 4 days. Every 27 days, a cycle will be repeated. OLCI will be upgraded in comparison to MERIS from 15 to 21 spectral channels in the wavelength range of 0.4 to $1.02 \mu \mathrm{m}^{1}$. The planned channels in the nearinfrared, relevant for water vapour retrievals, are shown in Fig. 1.

\subsection{METimage}

METimage will be a multispectral radiometer, mounted on the second generation of EUMETSAT Polar SYSTEM (EPSSG) satellites. This framework consists of 3 polar orbiting MetOp satellites and its prime objective is to provide continuous, long-term data sets of meteorological quantities. Metop-A was launched in 2006, Metop-B in 2012 and Metop-C will follow in 2016. The center of the METimage concept is a rotating telescope developed by Jena-Optik. The Visual/Infrared Imager (VII) will detect sunlight, reflected by the Earth, in the visible and near infrared spectrum from 0.4 to $14 \mu \mathrm{m}$. A spatial resolution of maximum $250 \mathrm{~m}$ and a field of view of $110^{\circ}$ (swath approximately $2800 \mathrm{~km}$ ) is expected. The radiometer scans 20 pixel simultaneously with a field of view of $1^{\circ}(20 \mathrm{~km})^{2}$. The channel constellation and the number of channels has not yet been finally decided between a 20-channel or a 30-channel concept. The Institute for Space Science at the Free University Berlin was asked to ascertain the best channel position and combination for the water vapour remote sensing. The planned channels in the near-infrared, relevant for water vapour retrievals, are shown in Fig. 2.

\subsection{MTG-FCI}

The Meteosat Third Generation (MTG) system is going to replace the Meteosat Second Generation (MSG). The concept of geostationary satellites is based on two three-axisstabilized platforms, the MTG-I (imager) and the MTG-S (sounder). The scheduled launch will be in 2020. A spectrometer will be installed on MTG-I, the Flexible Combined Imager (FCI). It can detect the electromagnetic radiation in 16 channels rather than 12 on MSG between 0.4 and $13.3 \mu \mathrm{m}$ with an expected spatial resolution of maximal $1 \mathrm{~km}$ between 0.4 and $2.1 \mu \mathrm{m}$ and a spatial resolution of $2 \mathrm{~km}$ between 3.8 and $13.3 \mu \mathrm{m}^{3}$. The planned channels in the near-infrared, relevant for water vapour retrievals, are shown in Fig. 3.

\section{Theory}

\subsection{Radiative transfer calculations}

In this study the radiative transfer model MOMO was used for the radiative transfer calculations in order to determine

\footnotetext{
${ }^{1}$ http://www.ioccg.org/sensors/olci.html.

${ }^{2}$ http://www.eumetsat.int.

${ }^{3} \mathrm{http}: / /$ www.eumetsat.int.
} 


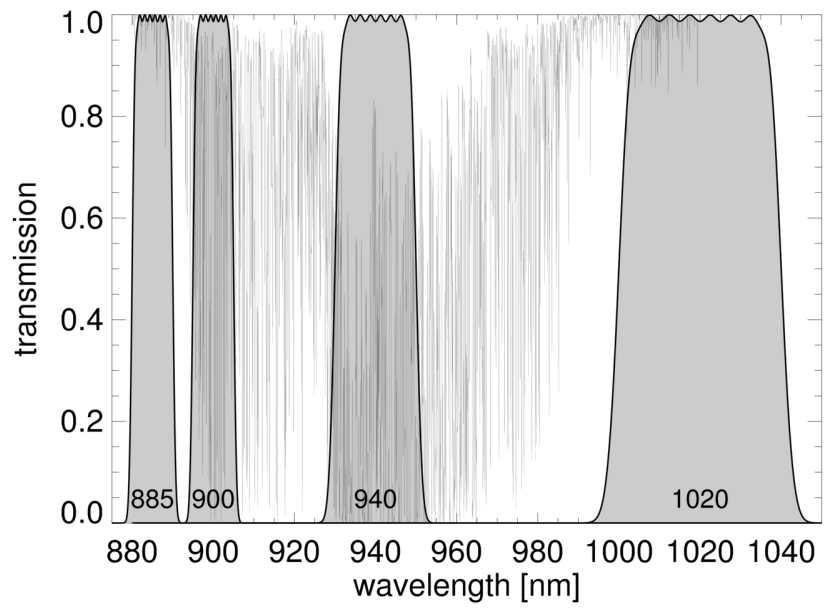

Fig. 1. OLCI channel constellation in the NIR (normalized response functions) and total transmittance of the atmosphere in $\rho \sigma \tau$-band.

the sensitivities of certain channels to different atmospheric properties. It is based on the Matrix Operator Method (Plass et al., 1973; Grant and Hunt, 1969a,b; Twomey et al., 1966). MOMO is a one-dimensional model with a coupled oceanatmosphere system. It derives the upward and downward radiative fluxes on the borders of the prior-defined vertical layers for selectable zenith- and azimuth-angles (Fischer and Grassl, 1984; Hollstein and Fischer, 2012). For this investigation, radiative transfer calculations have been performed for a part of the $\rho \sigma \tau$-band in the near infrared from 880 to $960 \mathrm{~nm}$. The scattering properties of different atmospheric components like ice clouds or aerosols were calculated using a Mie code based on Wiscombe (1980). The absorption coefficients of the atmospheric gases (mainly $\mathrm{H}_{2} \mathrm{O}$ and $\mathrm{O}_{2}$ ) were computed with the k-distribution method (Bennartz and Fischer, 2000). Information about the location and width of the absorption lines originate from the HITRAN database (Rothman et al., 2010).

\subsection{Quantification of uncertainies using optimal estimation}

\subsubsection{Best estimate error}

Any measurement $(\boldsymbol{y})$ has an associated uncertainty $\left(\sigma_{y}\right)$. In case of remote sensing, measurements are generally TOA radiances. The determination of the measurement error of the instrument is realizable by calibration and comparison measurements. Most techniques retrieve physical properties from radiance measurements through the use of sophisticated inversions. This means that the retrieved quantities (combined in the state vector $\boldsymbol{x})$ will also be uncertain $\left(\sigma_{x}\right.$ is the error of the retrieved quantity). These inversions commonly iteratively optimize the result of a forward model $\boldsymbol{F}$, which uses considerable additional (prior-)information (model parameters $\boldsymbol{p}$ ) that are also contaminated with errors $\sigma_{p}$ :

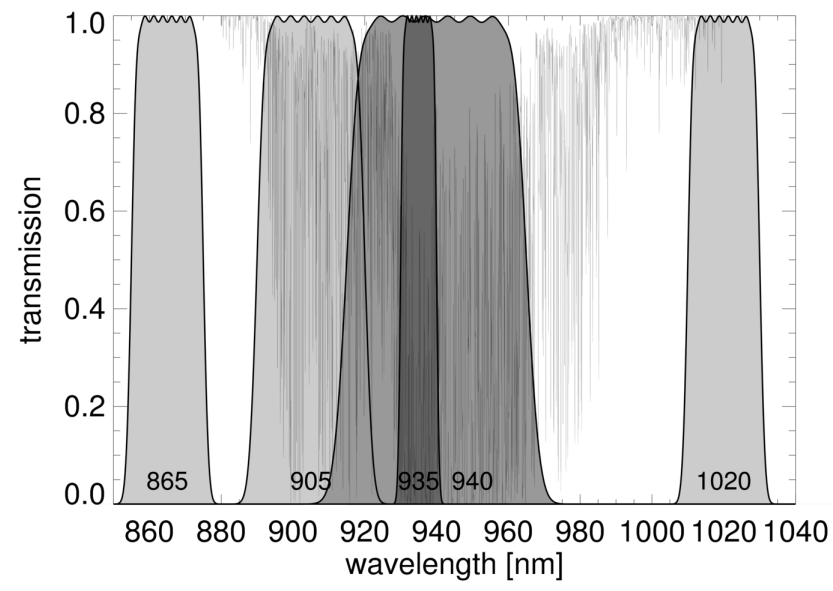

Fig. 2. METimage original channel constellation in the NIR (normalized response functions) and total transmittance of the atmosphere in $\rho \sigma \tau$-band.

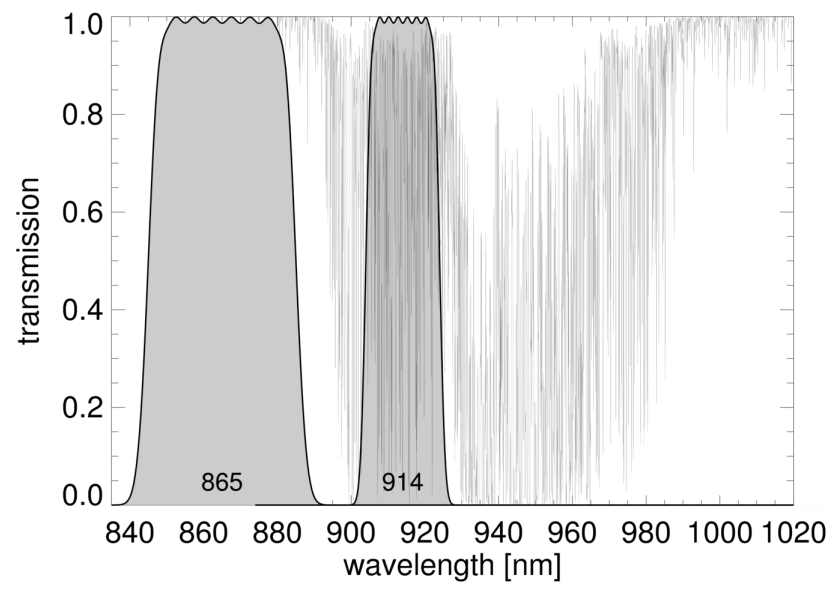

Fig. 3. MTG-FCI original channel constellation in the NIR (normalized response functions) and total transmittance of the atmosphere in $\rho \sigma \tau$-band.

$\boldsymbol{y} \pm \boldsymbol{\sigma}_{y}=\boldsymbol{F}\left(\boldsymbol{x} \pm \boldsymbol{\sigma}_{x}, \boldsymbol{p} \pm \boldsymbol{\sigma}_{p}\right)$.

As an example, the TOA radiance reflected from the Earth depends on parameters such as the surface albedo, the aerosol load, the water vapour content, and clouds, the temperature of each which can be measured only to a certain accuracy. The question is, how can we determine the uncertainty of the retrieved atmospheric property $\sigma_{x}$ without knowledge of the exact retrieval technique? One method was introduced by Rodgers (2000), and uses the Bayes' with an assumption of normally distributed errors. An overview of the method is presented here; for detailed derivation see the book by Rodgers (2000).

The influence of an individual parameter on the retrieved state can be calculated, assuming that the sensitivity of the 
measurement to the parameter is known. For example, in cases where the uncertainty of a model parameter is large but the sensitivity of the measurement to the parameter is negligible, there is no significant increase in the uncertainty of the retrieved state. The influence of individual parameters on the measurement can be simulated with a radiative transfer model (e.g. MOMO). The Jacobian of the radiance according to a certain parameter can be approximated by the change in radiance in response to a small change in the parameter, while keeping all other parameters constant.

A matrix $\mathbf{K}^{p}$ is constructed, which contains information about the sensitivity of the parameters to the measurement and whose size depends on the number of channels and parameters. The entries in the matrix are effectively the derivatives of the radiance in a channel $i$ to the variation of the $j$ model parameter:

$\mathbf{K}_{i, j}^{p}=\left\{\frac{\delta y_{i}}{\delta p_{j}}\right\}$.

In this case, local linearity must be assumed. The uncertainty of the prior knowledge of each model parameter is stored in a covariance matrix $\mathbf{S}^{p}$ :

$\mathbf{S}_{i, j}^{p}=\left\{c_{i, j} \sigma_{p, i} \sigma_{p, j}\right\}$,

where $\sigma_{p, i}$ is the uncertainty in units of standard deviation of the $i$ model parameter and $c_{i, j}$ is the linear correlation coefficient between the $i$ and $j$ parameter. For many applications this correlation is zero, resulting in a diagonal matrix $\mathbf{S}^{p}$. The measurement error covariance matrix $\mathbf{S}^{y}$ is defined as

$\mathbf{S}_{i, j}^{y}=\left\{c_{i, j} \sigma_{y, i} \sigma_{y, j}\right\}$,

where $\sigma_{y, i}$ is the measurement error in units of standard deviation for the $i$ channel of the spectrometer and $c_{i, j}$ is the correlation between the error in the $i$ and $j$ channel, which is zero for standard spectrometer. So $\mathbf{S}^{y}$ has the diagonal elements:

$\left(\sigma_{i, i}^{y}\right)^{2}=\left(L_{i} / \mathrm{SNR}_{i}\right)^{2}+\Delta_{i}^{2}$,

where $L_{i}$ is the radiance measured in channel $i, \mathrm{SNR}_{i}$ the signal to noise ratio $\left(\mathrm{SNR}=y / \sigma_{y}\right)$ and $\Delta_{i}$ the uncertainty of the absolute calibration of channel $i$. The matrix $\mathbf{K}$ carries information about the sensitivity of the measurement in the $i$ channel to the $j$ element of the state vector $\boldsymbol{x}$, so the derivatives of radiance to the retrieval quantity are

$\mathbf{K}_{i, j}=\left\{\frac{\delta y_{i}}{\delta x_{j}}\right\}$.

Finally, the error covariance matrix of the state vector is defined by

$\mathbf{S}^{x}=\left\{c_{i, j} \sigma_{x, i} \sigma_{x, j}\right\}$.
This matrix describes the uncertainties of the retrieved quantity. The square route of the diagonal elements of $\mathbf{S}^{x}$ specifies the error in the unit of the retrieved value, and is thus the sought after quantity

According to Rodgers (2000), the approach to calculate $\mathbf{S}^{x}$ is as follows. First, the model uncertainty $\mathbf{S}^{e}$ is derived by propagating the model parameter uncertainty $\mathbf{S}^{p}$ into the measurement space, and adding the measurement uncertainty $\mathbf{S}^{y}$ :

$\mathbf{S}^{e}=\mathbf{S}^{y}+\mathbf{K}^{p} S^{p} \mathbf{K}^{p^{T}}$.

The second step is the propagation of $\mathbf{S}^{e}$ into the state vector space, which is different from standard error propagation. The inverse model uncertainty is combined with the Jacobian of the forward operator $(\mathbf{K})$ :

$\mathbf{S}^{x}=\left(\mathbf{K}^{T} S^{e^{-1}} \mathbf{K}\right)^{-1}$.

Prior or background knowledge of the retrieval quantity is not included in this approach.

\subsubsection{Information content}

Another way of evaluating the retrieval error is the information content (Shannon and Weaver, 1949), which is analogous to the concept of entropy in thermodynamics. The information content $H$ provides the "value" of a measurement. It is the reduction in entropy $S$ :

$H=S\left(P_{1}\right)-S\left(P_{2}\right)$,

where $P_{1}$ is the uncertainty before and $P_{2}$ after the measurement. For a Gaussian distribution, the information content is calculated by Eq. (11):

$H=0.5 \log _{2}\left(\sigma_{a}^{2}\right)-0.5 \log _{2}\left(\sigma_{x}^{2}\right)$,

where $\sigma_{a}$ is the standard deviation due to the natural variability of the parameter and $\sigma_{x}$ is the uncertainty of the retrieved parameter. $H$ determines the number of possible states of the measurement in binary bits. For example, the water vapour column content normally varies between 1 and $70 \mathrm{~mm}$. If the retrieval can only derive the water vapour content with an accuracy of $10 \mathrm{~mm}, H$ is very low because the uncertanty is in the range of the natural variation. According to simple logarithm laws, Eq. (11) implies the quotient of the variances of the retrieval value and its uncertainty, which is similar to the concept of the signal-to-noise ratio. Thus, the higher the uncertainty of the retrieval, the lower the information content and the smaller the number of possible states that can be resolved based on the measurements.

\section{Atmospheric cases and assumptions}

In this work, the quantification of uncertainties was performed for a number of different cases. As already mentioned, the ratio of TOA radiances from an absorption and 
a window channel $\left(L_{\mathrm{abs}}, L_{\mathrm{win}}\right)$ provides information about the TCWV and is hereafter referred to as the apparent transmittance $R$ :

$R=L_{\text {abs }} / L_{\text {win }}$.

In the NIR spectrum, the apparent transmittance is most sensitive to the water vapour column content and is influenced mainly by the surface albedo and the aerosol optical thickness. Consequently, three cases were considered for each parameter (low, medium and high values) (see Table 1). This results in a total number of 27 atmospheric cases.

Additionally, three other model parameter sensitivities were determined: the influence of a cirrus cloud (varying the cirrus optical depth between 0.1 and 1.6), the change of the temperature profile (shifting the temperature profile by a constant offset of $\pm 10 \mathrm{~K}$ ), and the scale height of an aerosol layer (varying the scale height between $1000 \mathrm{~m}$ and $4000 \mathrm{~m}$, homogeneous aerosol layer with $500 \mathrm{~m}$ thickness).

The theory of optimal estimation requires the knowledge of the uncertainties of the model parameters. The assumed accuracies are given in Table 2 as standard deviations. These parameters were considered to account for the error influences on the TCWV. The sensitivity to cirrus clouds was considered to include the influence of sub-pixel cloud contamination. Also, present cloud detection algorithms are not accurate enough to detect thin clouds. The height of the aerosol layer (see below) and the natural change in the temperature profile are significant error influences. The uncertainties of the parameters were assumed. For the aerosol optical thickness, cirrus optical depth, aerosol scale height, and temperature profile, the accuracies were kept constant for each of the 27 cases. For the surface albedo, Carrer et al. (2010) was taken into account. This validation report is very comprehensive and includes a large number of results for the uncertainties of the albedo. The inter-comparisons between different procedures show a spread between 0.02 and 0.05 . On the one hand, the uncertainties were determined as constant with higher albedo, but on the other hand, constant in percentage, depending on the assumption regarding the bidirectional reflectance distribution function. In this study, an increasing error with larger surface albedo was assumed (see Table 2). However, the exact values do not influence the general outcome of this study.

Furthermore, the influences of the profile shape of water vapour, aerosol or temperature were not considered in the determination of the uncertainties. Again, only the temperature profile was altered equally in every layer. The US Standard atmosphere profile was used. The calculated uncertainties refer only to the whole vertical column of the atmosphere. Nevertheless, the profile shape plays an important role when interpreting the influences of the albedo and aerosol optical thickness. The peak altitude of the sensitivity for the water vapour column at the TOA can be understood from Fig. 4. Here, the sensitivities of the apparent transmittance $R$ (window channel at $885 \mathrm{~nm}$, absorption channel at $900 \mathrm{~nm}$ ) is

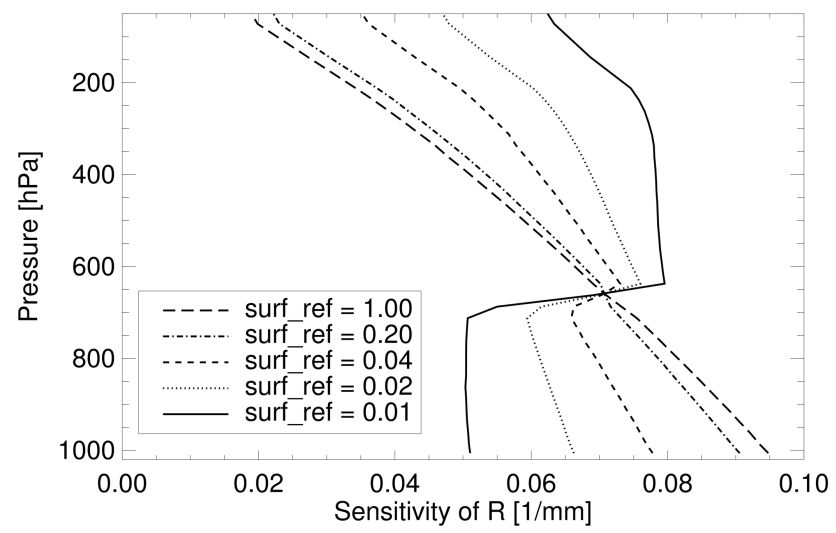

Fig. 4. Sensitivity in $1 \mathrm{~mm}$ of the apparent transmittance $R$ to the water vapour column content as a function of pressure (height levels) for five different surface reflectances (surf_ref). A constant aerosol layer at $700 \mathrm{hPa}$ is present.

Table 1. Sensitivity simulations input parameter cases.

\begin{tabular}{llll}
\hline Property & low & medium & high \\
\hline Water vapour column & $1 \mathrm{~mm}$ & $30 \mathrm{~mm}$ & $70 \mathrm{~mm}$ \\
Aerosol optical thickness & 0.1 & 0.4 & 1.0 \\
Surface albedo & 0.05 & 0.2 & 0.8 \\
\hline
\end{tabular}

shown as a function of height for five different surface reflectances. The sensitivities for each layer were derived from the difference between the reference ratio and the ratio simulated with an increased water vapour content $(+0.1 \mathrm{~mm})$ in that layer divided by the change in water vapour. For reference, the US standard water vapour profile was chosen and a homogeneous continental aerosol layer at $700 \mathrm{hPa}$ $(3000 \pm 250 \mathrm{~m})$ with an optical thickness at $900 \mathrm{~nm}$ of 0.1 . As the figure clearly shows, the peak altitude of the sensitivity is governed by the surface reflectance and the aerosol layer height. The lower the surface reflectance, the more information originates from the layer above the aerosol layer. The influence of the aerosol layer increases with higher optical thickness (not shown here). This is a significant error influence for surface reflectances below 0.2. Nevertheless, we only accounted for that in the parameter uncertainties (see Table 2).

The influence of other atmospheric properties, such as the Rayleigh optical thickness, carbon dioxide and amounts of other greenhouse gases, etc., was assumed to be very low in the wavelength range of interest. Consequently, these parameters were not regarded in this study and kept constant in the simulations. The aerosol optical thickness is highly dependent on the considered wavelength. In this work, the specified aerosol optical thicknesses are always defined at $900 \mathrm{~nm}$. In every case a continental aerosol was predefined. The size properties and refractive indices of the aerosol were taken from Hess et al. (1998). 
Table 2. Sensitivity simulations input parameter uncertainties in unit of standard deviation for each case (see Table 1).

\begin{tabular}{llll}
\hline Property & low & medium & high \\
\hline Aerosol optical thickness & 0.05 & 0.05 & 0.05 \\
Surface Albedo & 0.02 & 0.03 & 0.05 \\
\hline Cirrus optical depth & 0.1 & 0.1 & 0.1 \\
Aerosol scale height & $3000 \mathrm{~m}$ & $3000 \mathrm{~m}$ & $3000 \mathrm{~m}$ \\
Temperature profile & $20 \mathrm{~K}$ & $20 \mathrm{~K}$ & $20 \mathrm{~K}$ \\
\hline
\end{tabular}

The albedo of natural surfaces is a function of wavelength. In cases of real measurements, a common approach to account for this effect is to linearly extrapolate the surface reflectance in the window channels to estimate the surface reflectance in any spectrally close absorption channel. Within the framework of this study, the so-called albedo slope was not considered in the simulations, meaning that for a fixed case the same albedo was used for all simulated channels. However, when calculating the uncertainties, this phenomenon was included with the help of the following. The surface reflectance uncertainty affects the retrieval in two ways, the uncertainty of the absolute albedo and its spectral slope:

1. The uncertainty of the assumed surface reflectance in the window channels, resulting from uncertainties in the atmospheric correction, directly propagates to the uncertainty of the surface reflectance in the absorption channel. However, this error has the same sign in all channels, since the scattering properties hardly vary within the considered spectral ranges. Since the information used for the water vapour retrieval is provided by the ratio of absorption and window radiances, there is hardly any additional error contribution by the uncertain surface reflectance in the absorption channel. This is reflected by a high correlation of the uncertainties of both reflectance values in the error covariance matrix $\mathrm{S}_{\mathrm{e}}$.

2. The correlation of the surface reflectance uncertainties is reduced in cases of a strongly varying albedo slope, resulting in a higher error contribution by the absorption channel. A spectral surface albedo database was analysed with respect to the correlation of the surface reflectance values for different surface types and channel positions. Not surprisingly, the correlation is lower for spectrally distant channels, resulting in errors that are less correlated and higher retrieval uncertainties. The error covariance matrix $\mathbf{S}_{\mathrm{e}}$ of the model parameters (Eq. 12) was adapted accordingly.

Since the instruments are not built yet and calibration errors or signal-to-noise ratios (SNR) are not determined, the measurement errors can only be assumed (guided by the specifications of the concepts). For this reason, a standard SNR of 400 was preconditioned for each channel, which is in the approximate range of a NIR radiance measurement. The SNR is dependent on the channel width because more energy is coming in with a broader spectral range. However, this quantity turned out to have a non-significant influence and was kept constant. No absolute calibration uncertainties or systematic measurement errors were assumed.

Additionally, the instrument-specific response functions are not available yet. Nevertheless, the shape of response functions has a very low impact on the measured radiance. For convenience, the response function of the MERIS channel 15 was used. It was adapted to the different scheduled positions and widths (see Figs. 1, 2, 3). In the investigation of the optimal channel distribution, this response function was also used.

\section{Results}

\subsection{Uncertainties}

Uncertainties in mm of possible water vapour retrievals using the channel distributions of the original concepts of the three planned instruments are shown in Fig. 5. For OLCI and METimage, two window channels close to the $\rho \sigma \tau$-band were chosen. For MTG only one window and one absorption channel were considered. For the OLCI retrieval, two absorption channels, and for the METimage retrieval, three absorption channels, will be provided. For the positions and widths of the considered channels, see Sect. 2. The uncertainties of the 27 cases of a combination of three atmospheric properties are plotted. The legend for these cases is displayed below the plot as coloured bars of total water vapour column (TCWV) in blue, surface albedo (ALB) in yellow and aerosol optical thickness (AOT) in black. Although the uncertainties are discrete points, for better clarity a line was drawn between the points of each albedo class for sun zenith angle (SZA) of 0 degrees. Additionally, uncertainties for SZA of 20 and 60 degrees were calculated and plotted in dashed lines for each instrument.

The most striking feature is that the original METimage channel combination (in green) is more certain in almost every case (apart from dark surfaces with low water vapour content) than the others. The four-channel retrieval of OLCI in red has slightly larger uncertainties than METimage but less than the two-channel retrieval of MTG in purple (apart from cases with a bright surface and medium and high water vapour values). Generally, the uncertainties increase with higher water vapour content. This is due to the fact that absorption lines eventually reach saturation. In other words, the change of the transmittance in a water vapour absorption band approaches zero for higher values. Thus, sensitivity of the measurements to TCWV decreases with increasing humidity. 


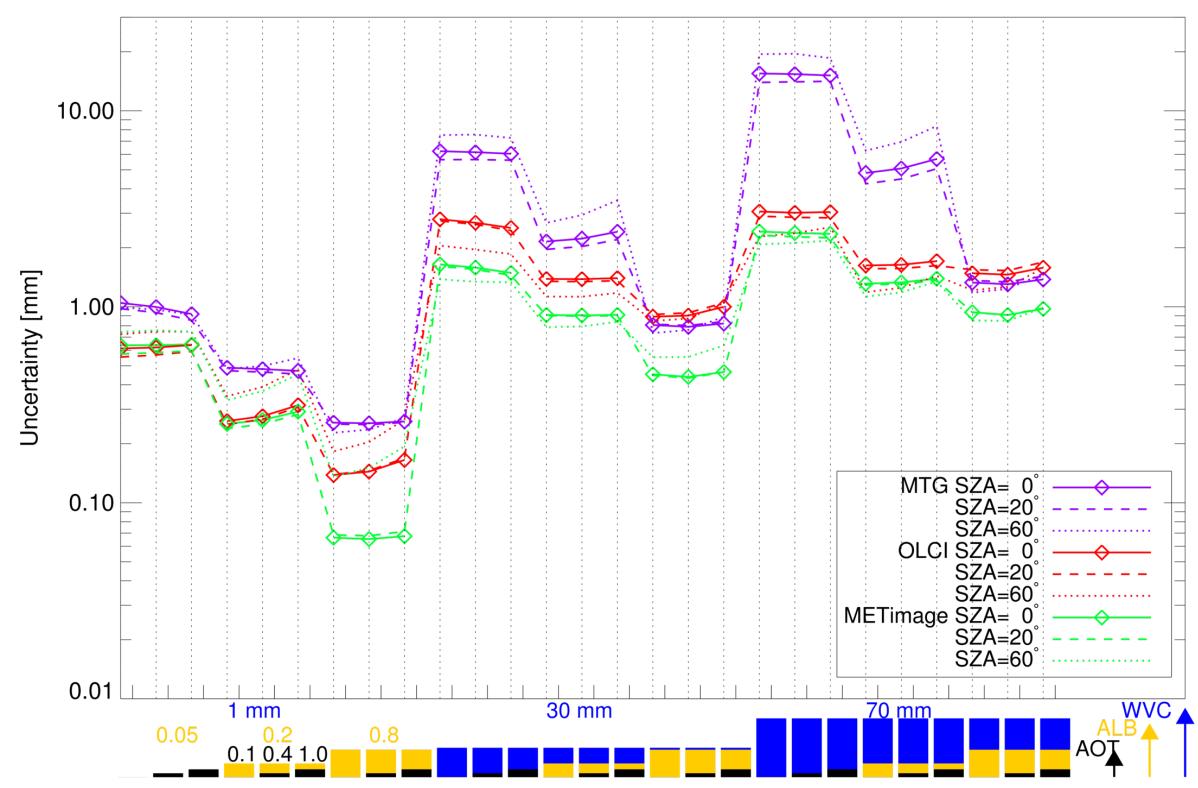

Fig. 5. Uncertainties of water vapour retrievals in $\mathrm{mm}$ of three channel combinations of future instruments (OLCI, METimage, MTG) for 27 different cases of a combination of three atmospheric properties: total column water vapour (TCWV), albedo (ALB), and aerosol optical thickness (AOT) - for three different sun zenith angles (SZA).

Another finding is the reduction of uncertainties with brighter surfaces, in some cases with one order of magnitude. Brighter surfaces reflect more radiation. Thus, the measurement is dominated by photons that have not been scattered at atmospheric particles. Above dark surfaces, a large number relative to the total amount of photons gets scattered back in the atmosphere and consequently does not travel through the whole vertical column of water vapour (Lindstrot et al., 2012) (see also Fig. 4). Hence, the uncertainty is higher for dark than for bright surfaces.

The dependency of the accuracy of the retrievals to the aerosol load is more dissimilar. In cases of a bright surface, the uncertainty slightly increases with a larger optical thickness of the aerosol layer. In contrast, in cases of a dark surface, the uncertainty slightly decreases with higher aerosol load. This is due to the fact that the aerosol layer, located in the lower troposphere, reflects a part of the radiation. Although this radiation does not reach the surface, the presence of the aerosol layer increases the fraction of measured photons that originate from the lower troposphere and thus decreases the error in the water vapour path (see Sect. 4 and Fig. 4).

To interpret the influence of the errors on the measurement, it is more enlightening to examine the uncertainties relative to the simulated amount of TCWV (not shown here). Generally, the relative uncertainties decrease with higher water vapour value. This is consistent because the sensors have limited measurement sensitivities. A very small change in transmittance due to a small TCWV amount can only be determined with low accuracy. In cases of MTG, the relative uncertainty exceeds $100 \%$; and in cases of METimage and OLCI, it is around $70 \%$ over dark surfaces. The lowest values can be examined over bright surfaces. Again, higher intensities provide more information for the sensor and therefore the uncertainties are smaller. For medium water vapour amounts around $30 \mathrm{~mm}$, one has to expect a relative error of around 1 to $10 \%$, depending on the surface and atmospheric circumstances.

To determine the influence of the different viewing geometries, uncertainties for two exemplary sun zenith angles were calculated and plotted. The impact of a SZA of 20 degrees on the accuracy is negligible. For dry cases, the uncertainties of all retrievals is higher at a SZA of 60 degrees than for a SZA of zero degrees. This applies also for MTG in the humid cases but not for OLCI and METimage.

\subsection{Retrievals with one and two absorption channels}

In the previous paragraph the behaviour of the scheduled channels was illustrated. The question arises as to whether this is the optimal distribution and combination of channels. As seen in Fig. 5, it seems that not only the location but also the number of absorption channels have an impact on the accuracy of the retrievals. MTG with only one absorption channel has larger uncertainties than the OLCI and METimage with two and three absorption channels.

In the following, the exemplary METimage channel locations were used in order to assume the optimal absorption channel. Figure 6 has the same format as Fig. 5. The original METimage retrieval is plotted in green again. The other colors account for one-absorption-channel retrievals with two 


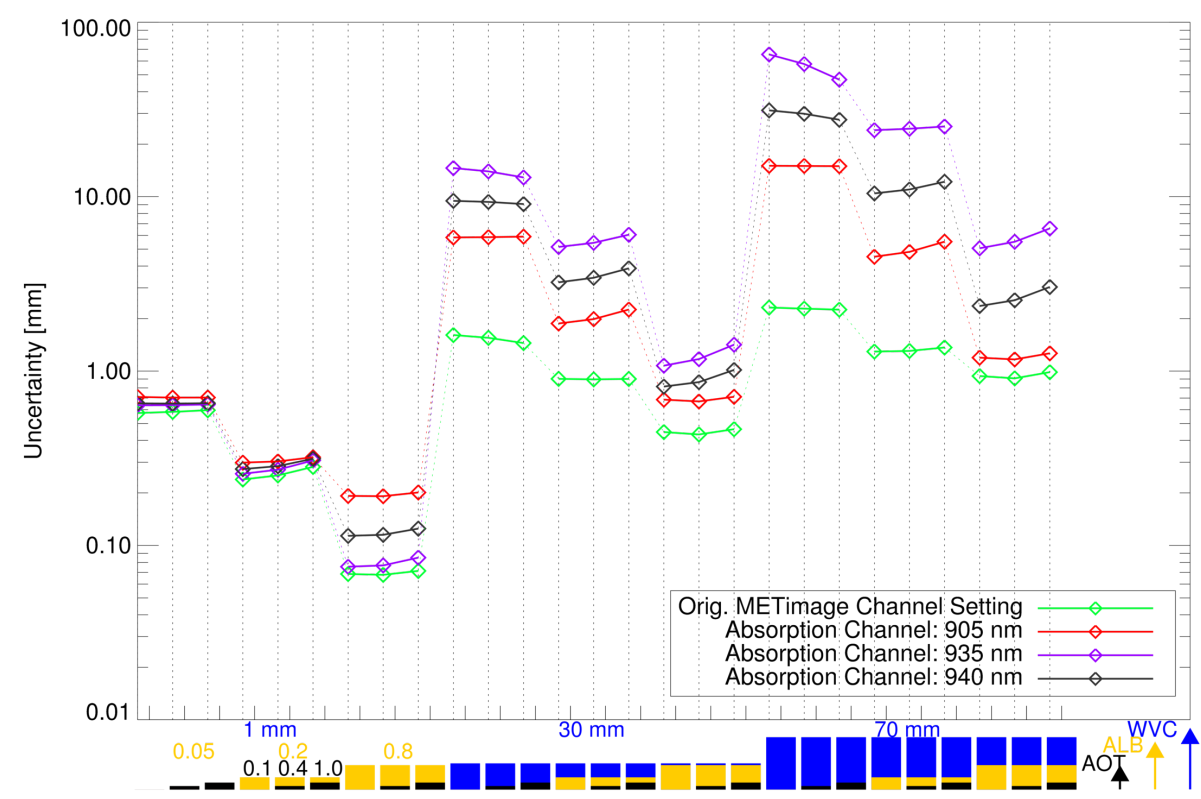

Fig. 6. Uncertainties in mm of water vapour retrievals using one absorption channel and two window channels (METimage concept) for 27 different cases of a combination of three atmospheric properties: total column water vapour (TCWV), albedo (ALB), and aerosol optical thickness (AOT).

window channels at 865 and 1020 and one of the three absorption channels, respectively $(905 \mathrm{~nm}$ in red, $935 \mathrm{~nm}$ in purple, $940 \mathrm{~nm}$ in dark grey). Obviously, the retrievals using only one absorption channel have larger uncertainties than the original METimage setup, in some (dark surface) cases even one order of magnitude. For cases with medium- and high water vapour, the lowest uncertainties were found for the retrieval using the absorption channel at $905 \mathrm{~nm}$. The other two have a very low accuracy, especially in the humid cases. This is due to the fact that they reach saturation more easily because they are located in a strong absorbing wavelength range. However, this behaviour benefits their accuracy in the dry cases. For low water vapour contents, the uncertainties are quite similar. Here, the retrievals with the absorption channels 935 and 940 have lower uncertainties.

Figure 7 shows the results of the uncertainty calculation of retrievals with two window channels and a combination of two absorption channels (see the legend on the right bottom of the plot). Again, the original METimage retrieval is plotted in green. The most evident feature in comparison to Fig. 6 is that the uncertainties are lower (see the scale) in nearly all cases and close to the retrievals using all scheduled channels. The one with the best accuracy is the combination of absorption channel at $905 \mathrm{~nm}$ and $935 \mathrm{~nm}$ because the difference to the original retrieval is very low in every case. However, the combination of absorption channel at 905 and $940 \mathrm{~nm}$ is best in cases of bright surfaces. In dry cases, the different combinations only differ minimally. In medium and humid cases, the combination of $935 \mathrm{~nm}$ and $940 \mathrm{~nm}$ (the strong absorbing channels) is the poorest. These latter medium and humid cases also seem to have the strongest sensitivity to the aerosol load.

In summary, retrievals with two absorption channels are more accurate than with only one. The best combination seems to be $905 \mathrm{~nm}$ and $935 \mathrm{~nm}$; it is nearly as certain as the original combination of all three absorption channels. The combination of a channel close to the first window channel and one in the strong absorbing range appears to be a good choice.

\subsection{Optimal channel setting}

In the following, our aim is to ascertain whether the channel positions, widths, and their combinations can be improved in order to decrease the uncertainties of a potential TCWV retrieval. For this purpose it is more convenient to examine the information content because it is similar to the relative error and indicates the quality of a measurement on a linear scale. The example shown is for the METimage build-up.

\subsubsection{One absorption channel retrieval}

First, the optimal location for a one absorption channel retrieval was sought. To this end, the information content for a retrieval with two window channels $(865$ and $1020 \mathrm{~nm})$ and one variable absorption channel with a width of $10 \mathrm{~nm}$ was calculated. Figure 8 shows the information content as a function of the wavelength (ordinate) and the cases of the atmospheric state, which are equivalent to the ones in the last paragraph (abscissa). Each pixel represents the information content of a certain case and a certain center wavelength. 


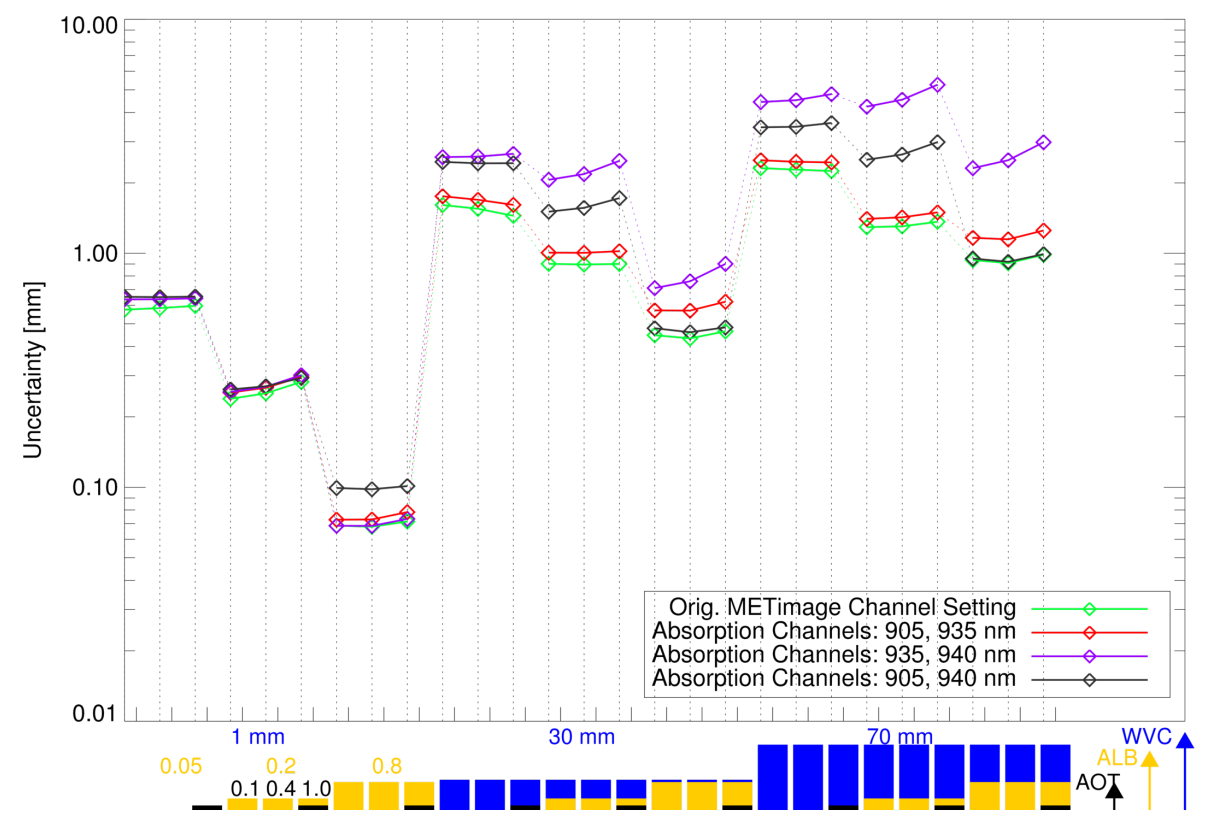

Fig. 7. Uncertainties in mm of water vapour retrievals using two absorption channels and two window channels (METimage concept) for 27 different cases of a combination of three atmospheric properties: total column water vapour (TCWV), albedo (ALB), and aerosol optical thickness (AOT).
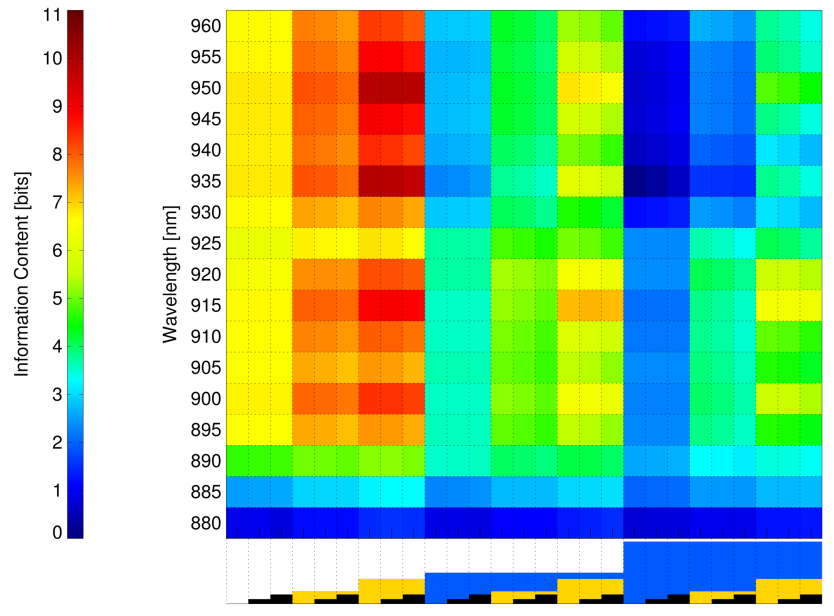

Fig. 8. Information content concerning water vapour of a retrieval using two window channels (METimage: 865, $1020 \mathrm{~nm}$ ) and one absorption channel (width $10 \mathrm{~nm}$, nadir view) for 27 cases of different atmospheric circumstances (abscissa with legend equivalent to Fig. 5).

Dark blue pixels account for low and red for high information content. Again, in short, the higher the information content, the higher the number of possible states that can be resolved and the lower the uncertainty of the model.

The most noticeable feature is again the decrement of information content with higher water vapour values. The strong connection between information content and surface albedo is also clear and is represented by the vertical bars of similar color. The highest information content (around 11 bits) was calculated in the dry cases at the strong absorbing channels represented by the deep red pixels. Reasons for that were mentioned in the last chapter. The lowest information content (represented by dark blue pixels) was determined for cases with a high water vapour content and in the strong absorbing wavelength range higher than $925 \mathrm{~nm}$. In changing atmospheric cases, the information content behaves as described before (apart from $880 \mathrm{~nm}$ and $885 \mathrm{~nm}$ ); only the magnitudes differ between the channels. If based exclusively on the findings from Fig. 8, it is difficult to decide which channel is the best for a water vapour retrieval. On the one hand, the maximum information content can be seen at $935 \mathrm{~nm}$ and $950 \mathrm{~nm}$, but only for dry cases. On the other hand, these give only a small amount information for humid cases. Hence, it is more convenient to analyse the information content averaged over all cases.

The mean, minimum and maximum information content of all cases as well as the standard deviation is displayed in Fig. 9 as a function of the simulated channels. As before, the strongly absorbing channels show the highest information content but at the same time provide almost no information for humid cases, which is contrary to the desired behaviour of a TCWV retrieval. The goal is to use an absorption channel which is least dependent on the atmospheric case. Looking at the mean, which is represented by the dashed line, two maxima appear at $900 \mathrm{~nm}$ and $915 \mathrm{~nm}$, both indicating a relatively high information content over all cases. The information content in these channels have a much smaller standard deviation than the other relative maximum at $950 \mathrm{~nm}$. This leads to the 


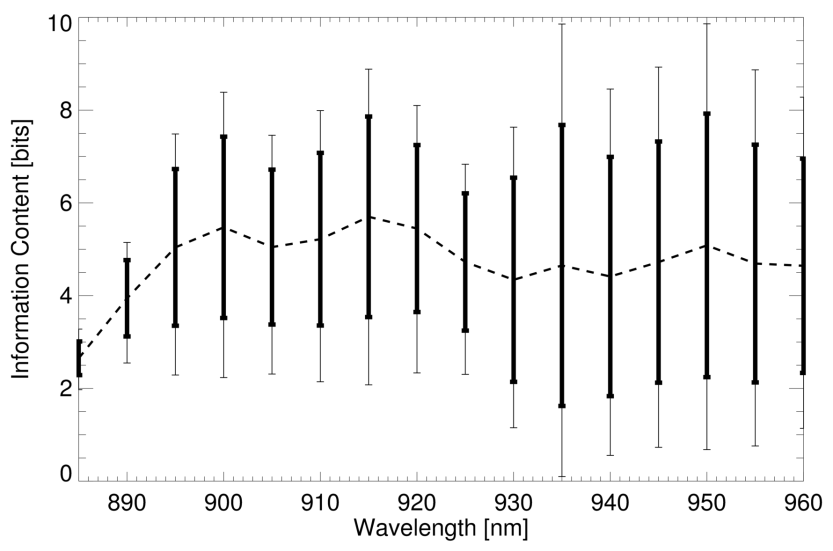

Fig. 9. Mean information content in 27 cases of different atmospheric circumstances concerning water vapour of a retrieval using two window channels (METimage: $865,1020 \mathrm{~nm}$ ) and an absorption channel (width $10 \mathrm{~nm}$ ) (broad bars account for the standard deviation of the cases).

conclusion that for a one absorption channel retrieval, either 900 or 915 can serve as the best choice and the MTG-FCI channel selection is optimal.

\subsubsection{Two absorption channel retrieval}

The information content of a two absorption channel retrieval is displayed in Fig. 10 with similar logic as in Fig. 9. One absorption channel is at $900 \mathrm{~nm}$ (width $10 \mathrm{~nm}$ ) and the other is at the location indicated at the ordinate (width again $10 \mathrm{~nm}$ ). Overall, the information content is increased in comparison to Fig. 9. The highest mean, maximum and minimum information contents were determined for wavelengths above $925 \mathrm{~nm}$. The maxima at 935 and $950 \mathrm{~nm}$ are outstanding (with a mean of 6.5 respectively 6 bits over all cases). At these wavelengths, the minimum and maximum information content is maximal as well. Thus, the optimal second absorption channel for this case is at $935 \mathrm{~nm}$.

The information content for a two absorption channel retrieval with a fixed first absorption channel at $915 \mathrm{~nm}$ is plotted in Fig. 11. Generally, the information contents are in the same range as in Fig. 10. Here, only one maximum in the mean information content at $950 \mathrm{~nm}$ is evident and is in the same range as the combination 900 and $935 \mathrm{~nm}$. Consequently, the combination of $915 \mathrm{~nm}$ and $950 \mathrm{~nm}$ is a good choice for a two absorption channel retrieval as well. Comparing Figs. 10 and 11 reveals that the combination of $900 \mathrm{~nm}$ and $935 \mathrm{~nm}$ gives slightly higher information contents than $915 \mathrm{~nm}$ and $950 \mathrm{~nm}$. The water vapour channels of METimage are therefore well designed. The $940 \mathrm{~nm}$ channel does not improve the retrieval uncertainties significantly (see Fig. 7). This is probably due to the fact that it is planned to have a width of $50 \mathrm{~nm}$ and therefore overlap with the $935 \mathrm{~nm}$ and $905 \mathrm{~nm}$ (see Fig. 2). Generally, a two absorption channel retrieval is more reliable if the absorption channels are least

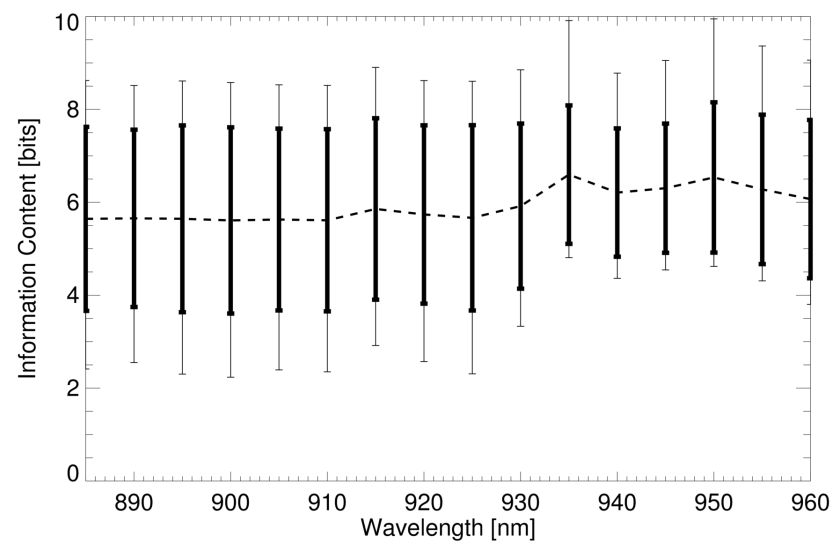

Fig. 10. Mean information content in 27 cases of different atmospheric circumstances concerning water vapour of a retrieval using two window channels $(865,1020 \mathrm{~nm})$, a fixed first absorption channel at $900 \mathrm{~nm}$ and a second absorption channel (width $10 \mathrm{~nm}$ ) (broad bars account for the standard deviation of the cases).

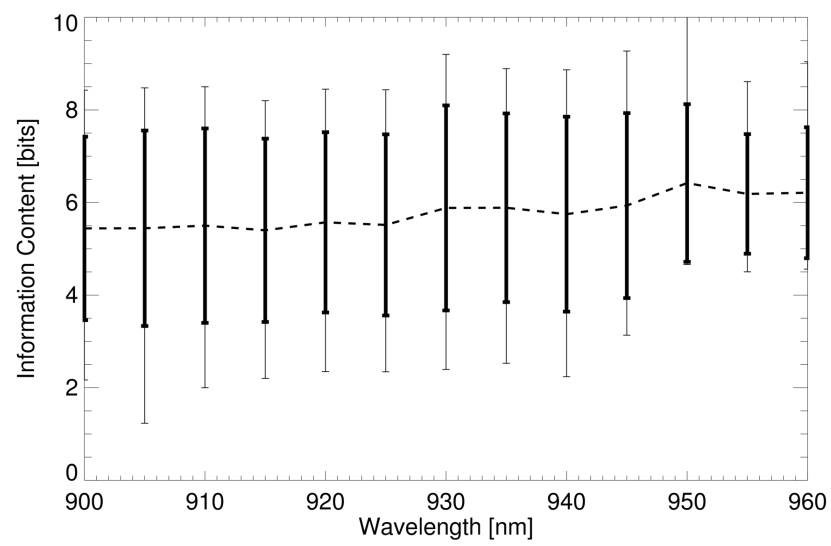

Fig. 11. Mean information content in 27 cases of different atmospheric circumstances concerning water vapour of a retrieval using two window channels $(865,1020 \mathrm{~nm})$, a fixed first absorption channel at $915 \mathrm{~nm}$ and a second absorption channel (width $10 \mathrm{~nm}$ ) (broad bars account for the standard deviation of the cases).

correlated. This was confirmed by cross-correlating all simulated radiances in the different channels (not shown). The lowest correlation coefficient was calculated for the combination of 900 and $935 \mathrm{~nm}$.

\section{Summary and conclusion}

This study analyses the behaviour and the quantities of the uncertainties of possible TCWV retrievals using TOA radiance measurements from future instruments. Depending on the atmospheric state, relative errors of up to $10 \%$ for medium water vapour values and up to $100 \%$ for very dry cases can be expected. The absolute uncertainties increase with higher water vapour content because of $\mathrm{H}_{2} \mathrm{O}$ saturation. 
Brighter surfaces reflect more radiation and thus the uncertainty decreases with higher surface albedo. The accuracy decreases slightly with higher optical thickness over medium and bright surfaces. In cases of a very dark surface, a thick aerosol layer can increase the accuracy slightly because of the increased amount of radiation that has been reflected in the lower troposphere. The overall uncertainty over all cases turns out to be a function of the number of considered absorption channels. The METimage channel setting with three absorption channels was more accurate than the OLCI setting with two absorption channels and the MTG-FCI with one absorption channel for most cases.

The determination of the optimal position of the absorption channels was done in two steps. First, a retrieval using one absorption channel and the instrument-specific window channels were used to calculate the information content for each atmospheric case. The case-dependent behaviour of the information content is similar to the characteristics of the uncertainties: the information content decreases with higher water vapour values (due to $\mathrm{H}_{2} \mathrm{O}$-saturation), increases with higher surface albedo, and decreases slightly with a higher aerosol load. However, there are differences in magnitude between the used absorption channels. The optimal position for the "first" absorption channel was determined to be at 900 or $915 \mathrm{~nm}$. The second step was to calculate the accuracy of a retrieval using the window channels, one fixed absorption channel and a second absorption channel with varying position. It turned out that the second absorption channel is optimally placed in the strong absorbing range above $930 \mathrm{~nm}$. The highest information contents were determined for the combination of $900 \mathrm{~nm}$ and $935 \mathrm{~nm}$. This was explained by the low correlation between theses wavelengths. The channel width, which had been varied between 10 and $20 \mathrm{~nm}$, did not seem to have a strong influence on the information content. A second absorption channel of $20 \mathrm{~nm}$ width slightly improved the accuracy of the retrievals (not shown). Consequences for the schedules of the instruments are comparably minor. For the METimage concept, the broad $940 \mathrm{~nm}$ absorption channel should not be considered. In the OLCI schedule, the location of the second absorption channel should be changed to $935 \mathrm{~nm}$. The $914 \mathrm{~nm}$ channel of the MTG-FCI concept seems to be a good choice. In general, though, many retrievals and applications already use the $900 \mathrm{~nm}$ to determine TCWV. To continue the time series of, e.g. MERIS on ENVISAT, with the same retrievals, the $900 \mathrm{~nm}$ channel is the recommended choice.

Edited by: P. Stammes

\section{References}

Albert, P., Bennartz, R., and Fischer, J.: Remote sensing of atmospheric water vapor from backscattered sunlight in cloudy atmospheres, J. Atmos. Ocean. Tech., 18, 865-874, doi:10.1175/1520-0426(2001)018<0865:RSOAWV>2.0.CO;2, 2001.

Albert, P., Bennartz, R., Preusker, R., Leinweber, R., and Fischer, J.: Remote sensing of atmospheric water vapor using the moderate resolution imaging spectroradiometer, J. Atmos. Ocean. Tech., 22, 309-314, doi:10.1175/JTECH1708.1, 2005.

Bartsch, B., Bakan, S., and Fischer, J.: Passive remote sensing of the atmospheric water vapour content above land surfaces, Adv. Space Res., 18, 25-28, doi:10.1016/0273-1177(95)002855, 1996.

Bennartz, R. and Fischer, J.: A modified k-distribution approach applied to narrow band water vapour and oxygen absorption estimates in the near infrared, J. Quant. Spectrosc. Ra., 66, 539-553, doi:10.1016/S0022-4073(99)00184-3, 2000.

Carrer, D., Roujean, J.-L., and Meurey, C.: Comparing Operational MSG/SEVIRI Land Surface Albedo Products From Land SAF With Ground Measurements and MODIS, IEEE T. Geosci. Remote, 48, 1714-1728, doi:10.1109/TGRS.2009.2034530, 2010.

Fischer, J. and Grassl, H.: Radiative transfer in an atmosphere-ocean system: an azimuthally dependent matrix-operator approach, Appl. Optics, 23, 1032-1039, doi:10.1364/AO.23.001032, 1984.

Fischer, J. and Leinweber, R. P. R.: Retrieval of total water vapour content from MERIS measurements algorithm theoretical basis document ATBD 2.4, Free University Berlin, Institute for Space Science, Berlin, 2010.

Gao, B.-C., Goetz, A. F. H., Westwater, E. R., Conel, J. E., and Green, R. O.: Possible near-IR channels for remote sensing precipitable water vapor from geostationary satellite platforms, J. Appl. Meteorol., 32, 1791-1801, doi:10.1175/15200450(1993)032<1791:PNICFR>2.0.CO;2, 1993.

Grant, I. P. and Hunt, G. E.: Discrete space theory of radiative transfer, I. Fundamentals, P. Roy. Soc. Lond. A Mat., 313, 183-197, 1969a.

Grant, I. P. and Hunt, G. E.: Discrete space theory of radiative transfer, II. Stability and non- negativity, P. Roy. Soc. Lond. A Mat., 313, 199-216, 1969b.

Hollstein, A. and Fischer, J.: Radiative transfer solutions for coupled atmosphere ocean systems using the matrix operator technique, J. Quant. Spectrosc. Ra., 113, 536-548, doi:10.1016/j.jqsrt.2012.01.010, 2012.

Hess, M., Koepke, P., and Schult, I.: Optical Properties of Aerosols and Clouds: The Software Package OPAC, B. Am. Meteorol. Soc., 79, 831-844, doi:10.1175/15200477(1998)079<0831:OPOAAC>2.0.CO;2, 1998.

Lindstrot, R., Preusker, R., Diedrich, H., Doppler, L., Bennartz, R., and Fischer, J.: 1D-Var retrieval of daytime total columnar water vapour from MERIS measurements, Atmos. Meas. Tech., 5, 631646, doi:10.5194/amt-5-631-2012, 2012.

Noël, S., Buchwitz, M., and Burrows, J. P.: Retrieval of total water vapour column amounts from GOME/ERS-2 data, Adv. Space Res., 29, 1697-1702, 2002.

Plass, G. N., Kattawar, G. W., and Catchings, F. E.: Matrix operator theory of radiative transfer, 1: Rayleigh scattering, Appl. Optics, 12, 314-329, doi:10.1364/AO.12.000314, 1973. 
Rodgers, C.: Inverse Methods for Atmospheric Sounding: Theory and Practice, World Scientific Pub Co., Oxford, 2000.

Rothman, L. S., Rinsland, C. P., Goldman, A., Massie, S. T., Edwards, D. P., Flaud, J.-M., Perrin, A., Camy-Peyret, C., Dana, V., Mandin, J.-Y., Schroeder, J., McCann, A., Gamache, R. R., Wattson, R. B., Yoshino, K., Chance, K., Jucks, K., Brown, L. R., Nemtchinov, V., and Varanasi, P.: Reprint of: The HITRAN molecular spectroscopic database and HAWKS (HITRAN Atmospheric Workstation): 1996 edition, J. Quant. Spectrosc. Ra., 111, 1568-1613, doi:10.1016/j.jqsrt.2010.04.019, 2010.

Schluessel, P. and Emery, W. J.: Atmospheric water vapour over oceans from SSM/I measurements, Int. J. Remote Sens., 11, 753 766, doi:10.1080/01431169008955055, 1990.

Shannon, C. E. and Weaver, W.: The Mathematical Theory of Communication, 1949.
IPCC: Climate Change 2007: The Physical Science Basis, Contribution of Working Group I to the Fourth Assessment Report of the Intergovernmental Panel on Climate Change, edited by: Solomon, S., Qin, D., Manning, M., Chen Z., Marquis, M., Averyt, K. B., Tignor, M., and Miller, H. L., 996 pp., Cambridge University Press, Cambridge, United Kingdom and New York, NY, USA, 2007.

Susskind, J., Barnet, C. D., and Blaisdell, J. M.: Retrieval of atmospheric and surface parameters from AIRS/AMSU/HSB data in the presence of clouds, IEEE T. Geosci. Remote, 41, 390-409, 2003.

Twomey, S., Jacobowitz, H., and Howell, H. B.: Matrix methods for multiple-scattering problems, J. Atmos. Sci., 23, 289-298, 1966.

Wiscombe, W. J.: Improved Mie scattering algorithms, Appl. Optics, 19, 1505-1509, doi:, 1980. 